\title{
Study on measurement method of radio waves generated by charged particles in cosmic-ray air showers using a particle accelerator
}

\author{
Y.Katayose $^{a}{ }^{*}$ T.Asaba $^{a}$, K.Honda $^{b}$, S.Kodaira, ${ }^{c}$ Y.Koshiba $^{d}$, K.Sakaue $^{e}$, T.Saito $^{b}$, \\ T.Sasaki $^{a}$, K.Takasaki $^{b}$, K. Yagisawa ${ }^{a}$ and M.Wakamatsu ${ }^{a}$ \\ a Faculty of Engineering, Yokohama National University \\ $b$ Tokyo Metro. Coll. Of Ind. Tech \\ c Nati. Inst. Rad. Sci., \\ $d$ Res. Inst. For Sci. and Engn., Waseda Univ., \\ $e$ Photon Science Center of the University of Tokyo
}

\begin{abstract}
To develop a detector with an effective area large enough to observe an extensive air shower, we studied a method of detecting radio waves generated via the transition radiation process by charged particles in an air shower. Characteristics of the radio waves were investigated using beam accelerators and radio antennas. When about $430 \times 10^{9}$ electrons with a kinetic energy of $4.7 \mathrm{MeV}$ crossed a titanium film into the air, the radio pulse emitted was measured using a standard dipole antenna with a bandwidth of $800-880 \mathrm{MHz}$. The measured pulse height at a position $1.2 \mathrm{~m}$ away from the source and at an angle of $5^{\circ}$ from the beam axis was approximately $670 \mathrm{mV}$. It was found that the electric field strength had large angular dependence and that the polarization property was roughly consistent with the theoretical value.
\end{abstract}

36th International Cosmic Ray Conference -ICRC2019-

July 24th - August 1st, 2019

Madison, WI, U.S.A.

\footnotetext{
*E-mail: katayose-yusaku-dv@ynu.ac.jp
} 


\section{Introduction}

For ultra-high-energy cosmic ray observation, a huge detector is required because the fluxes are extremely small. Direct observational of cosmic rays with energies greater than about $10^{15}$ electron volts is difficult, the only method is indirect measurement such as air shower observation or neutrino measurement with the earth as a part of the detector. One way to do the former is to use a large scale detector to measure radio waves emitted by charged particles in a cascade shower generated by primary particles. Several groups are making efforts to develop such a detector. For example, the ARA group has been attempting to measure of Cerenkov radio from a shower of particles originating from neutrinos traveling through ice at the South Pole [1]. Several other groups have also had great success in measuring radio waves from the air shower [2, 3]. However, when the results from such a radio-wave are compared to those obtained using a conventional detector, the performance is still insufficient. Thus, in order to increase performance, a better measuring technique needs to be developed. Radio waves are emitted from the air shower as synchrotron, bremsstrahlung, or Cerenkov radiation, and when charged particles that reach the ground invade the atmosphere, radio waves are also generated by the transition radiation processes. In this study, we use a particle accelerator to investigate the characteristics of radio waves generated by the transition radiation process. In the field of particle accelerator development, transition radiation is utilized for beam diagnostics. There are also studies to improve the position resolution using radiation in the EUV region and studies that use millimeter-long wavelengths in order to obtain larger coherence effects. Since air shower particles are scattered about a wide range from several tens of meters or more, longer wavelengths are better suited for obtaining sufficient coherence effects of radio waves from charged particles. In this study, we try to measure radio waves created by the transition radiation process in the range of tens of $\mathrm{MHz}$ to sub-GHz.

\subsection{Transition Radiation}

Transition radiation is emitted when a charged particle passes through inhomogeneous media. This can occur, for example, when a charged particle passes through a boundary between two different media. There are two different descriptions of the generation mechanism. One of the explanations is that the sudden appearance and sudden death signals are generated when charged particles collide with a substance. For example, the sudden appearance signal is seen during beam test experiments, for which the beam is hidden to the observer inside the accelerator and after which it "suddenly" becomes visible while exiting the accelerator pipe. An example of the sudden death signal is the signal originating from a beam-dump process. Ginzburg and Frank also explained transition radiation theoretically [4,5] by solving Maxwell's equations subject to boundary conditions. When a charged particle with charge q moves along the $z$-axis and traverses two substances each with a different dielectric constant and magnetic permeability, the electric field intensity radiated forward by the transition radiation process is given by the following equation [5, 6]:

$$
\left|\frac{d E(\omega)}{d \omega}\right|=\left|\frac{q \beta^{2}}{\pi v R} \sin \theta \cos \theta e^{i R \frac{\omega}{c} \sqrt{\chi_{2}}-i \omega t} \chi_{2}^{3 / 2} \xi_{2}\right|
$$


,where

$$
\xi_{2}=\frac{\left(\varepsilon_{1} / \varepsilon_{2}-1\right)\left(1-\beta \sqrt{\chi_{1}-\chi_{2} \sin ^{2} \theta}-\beta^{2} \chi_{2}\right)-\beta^{2} \varepsilon_{1} \mu_{2}\left(\mu_{1} / \mu_{2}-1\right)}{\left[\varepsilon_{2} \sqrt{\chi_{1}-\chi_{2} \sin ^{2} \theta}+\varepsilon_{1} \sqrt{\chi_{2}} \cos \theta\right]\left(1-\beta^{2} \chi_{2} \cos ^{2} \theta_{2}\right)\left(1-\beta \sqrt{\chi_{1}-\chi_{2} \sin ^{2} \theta}\right)}
$$

,$R$ is the distance from the point of entry into the boundary to an observation point, $v$ is the particle velocity, $\varepsilon_{1,2}$ and $\mu_{1,2}$ are the dielectric constant and magnetic permeability, respectively. $\chi_{2}=\varepsilon_{2} \mu_{2}$ and $\theta$ is the angle between the $z$-axis and the wave number vector. In this study, we evaluate the experimental results using equation 1.1

\section{Experiment and Results}

We investigated the validity of the transition radiation model using a well-characterized charged particles produced by a particle accelerator.

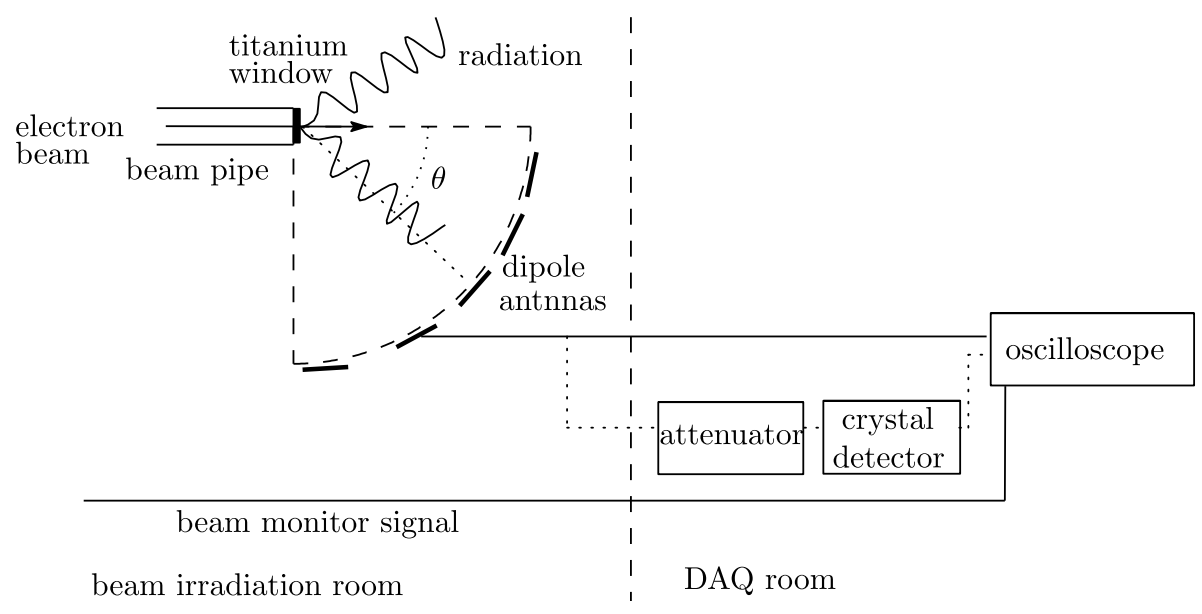

Figure 1: Setup of the electron irradiation experiment

\subsection{Experiment Using a Sub-GHz-band Antenna}

A compact antenna of sub-GHz band was used, and radio waves emitted from electrons passing from the titanium film to the air were measured. A photo cathode (RF-Gun) type electron beam accelerator of Waseda University was used. The accelerator produced electrons with a kinetic energy of $4.7 \mathrm{MeV}$. One bunch included five pulses, the pulse interval was $8.4 \mathrm{~ns}$, and the bunch interval was $200 \mathrm{~ms}(5 \mathrm{~Hz})$. The pulse width was $3 \mathrm{ps}$, the RMS size was $5 \mathrm{~mm}$, and approximately $700 \mathrm{pC}$ of charge (from electrons) was contained in this pulse. A standard dipole antenna (Anritsu MA5612A1) with a bandwidth of $800-880 \mathrm{MHz}$ was installed at a distance of $1.2 \mathrm{~m}$ from the titanium film window located at the end of the beam pipe as shown in the Fig. 1. The output signal was transmitted to the monitor room by a 20 -m coaxial cable (RG58/U), and the signal waveform was measured using an oscilloscope with an analog bandwidth of $1 \mathrm{GHz}$ (Tektronix TDS784D ). 

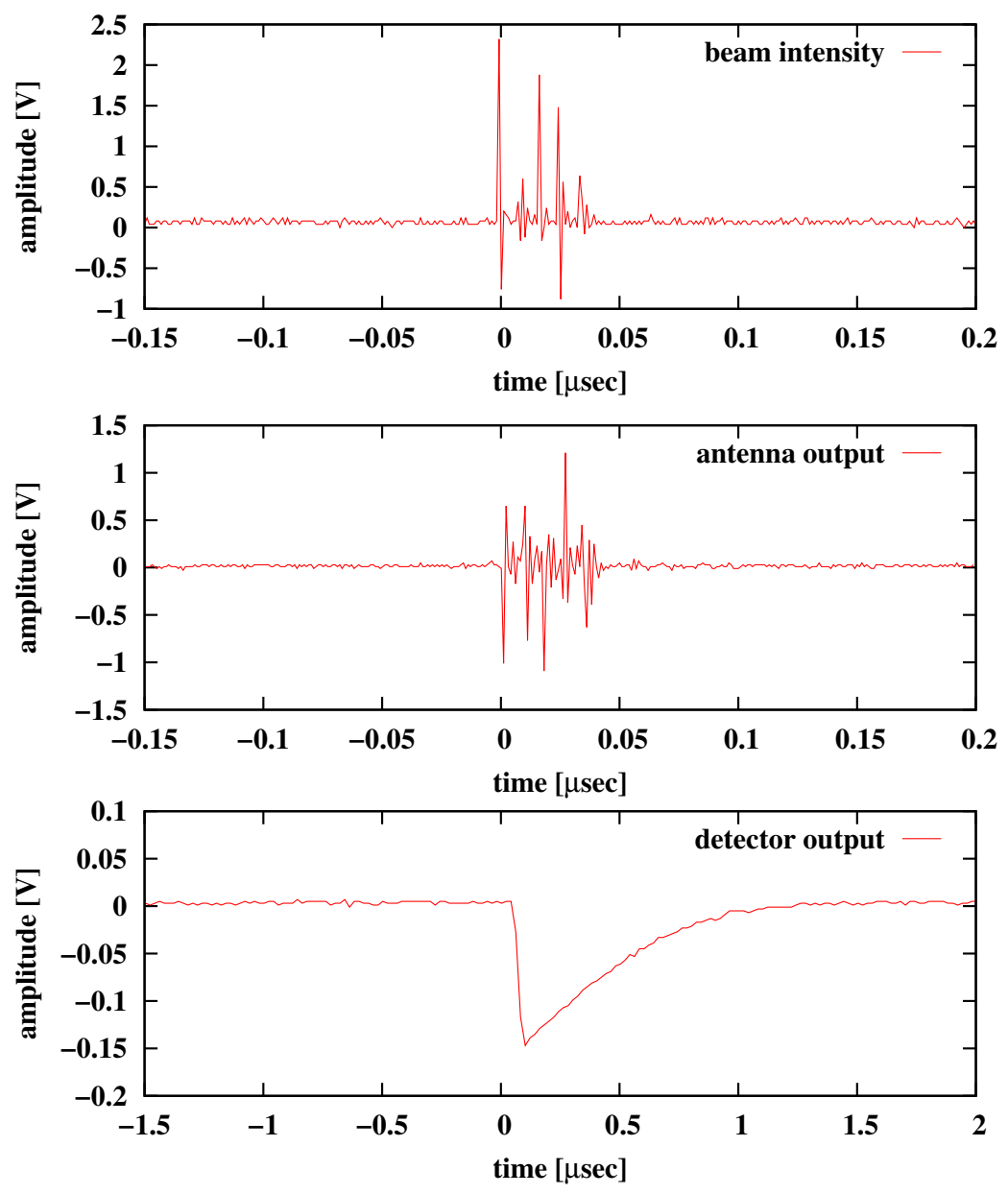

Figure 2: Waveforms of signals. The top diagram shows the beam intensity monitor. The middle diagram shows the raw signal of the antenna, and the bottom diagram shows the output signal of the crystal detector.

\section{$\underline{\text { Results }}$}

As shown in the middle diagram of Fig. 2, five consecutive sharp bipolar pulses synchronized with the beam were measured. The average value of the amplitudes of the five signals was about $670 \mathrm{mV}$. The top diagram of Fig. 2 shows the waveform of the beam intensity monitor. The peak value is proportional to the beam intensity, and $1 \mathrm{~V}$ corresponds to about $500 \mathrm{pC}$. The electric field strength received by the antenna is converted to voltage pulse strength by the following integration:

$$
V_{\text {out }}=\int\left(\frac{d E}{d \omega}\right)_{T r} \times n_{e} \times A_{f}(\omega) \times C_{a}(\omega) d \omega
$$

,where $n_{e}$ is the electron number per beam pulse, $A_{f}$ is an antenna factor and $C_{a}$ is a cable attenuation factor. The attenuation factor of RG58/U was approximately $700 \mathrm{~dB} / \mathrm{km}$ at a frequency of $800 \mathrm{MHz}$. Assuming that the dielectric constant of titanium was about 100, the integral value in the range of $500 \mathrm{MHz} \sim 3 \mathrm{GHz}$ was approximately $1144 \mathrm{mV}$, and the experimental values were roughly consistent with the calculated values.

In order to investigate the direction dependency of the transition radiation intensity, radio waves 


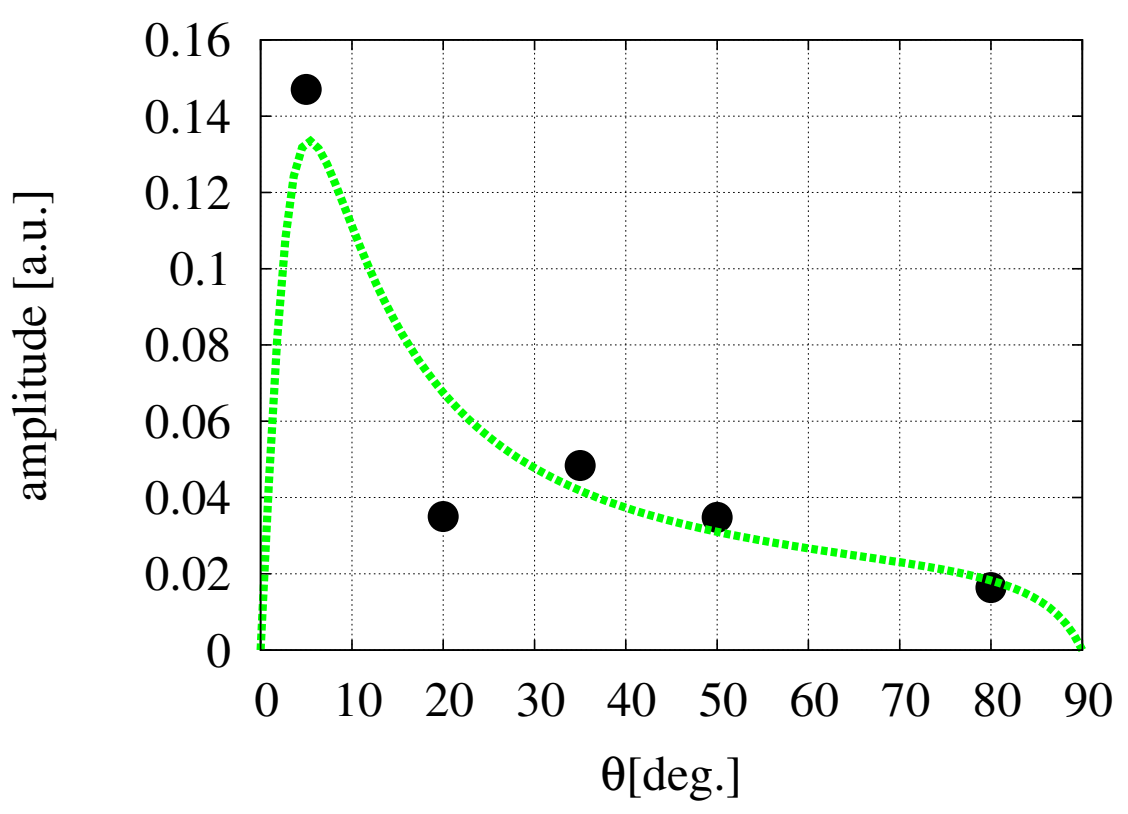

Figure 3: Angular dependence of electric field strength. The dashed line is the result of fitting using equation 1.1

were measured with five antennas installed at positions of $5^{\circ}, 20^{\circ}, 35^{\circ}, 50^{\circ}$, and $80^{\circ}$ as shown in Fig. 1. The antenna element was placed at the same height as the beam axis, and the direction of antenna element was set perpendicular to the vector connecting the measurement point from the beam window. In addition, in order to improve the measurement accuracy, a crystal detector was used to convert the raw signal pulse into a pulse with a large time constant. An attenuator was also used to prevent signal saturation in the detector. The bottom diagram of Fig. 2 shows the output signal of the crystal detector. The angular distribution of intensity is shown in Fig. 3. The intensity is largest at $5^{\circ}$, and the amplitude at $20^{\circ}$ or more is about one third of the amplitude at $5^{\circ}$. The dashed line is the result of fitting using equation 1.1. At an angle of $5^{\circ}$, the signal is lager than expected value from the theory. This can be caused by other factors, such as Coulomb field effect related to charge.

In order to confirm the polarization characteristics of the transition radiation, radio waves were measured while rotating the element direction of the dipole antenna. In Fig. 4, $\varphi$ is an angle of the direction of the antenna element in a plane perpendicular to the axis connecting the antenna from the beam window. It is expected that the signal will be strongest at $0^{\circ}$ and that the electric field strength will be proportional to $\cos (\varphi)$. The dashed line is a cosine function, and it was confirmed that the polarization was radial.

\subsection{Experiment Using a Wide-band Antenna}

Better coherence effects are easily obtained by measurement at frequencies of several tens of $\mathrm{MHz}$ to several hundreds of MHz. As the size of the antenna increase, the frequency decreases, and so, we used the HIMAC accelerator of the National Institute of Radiological Science, which 


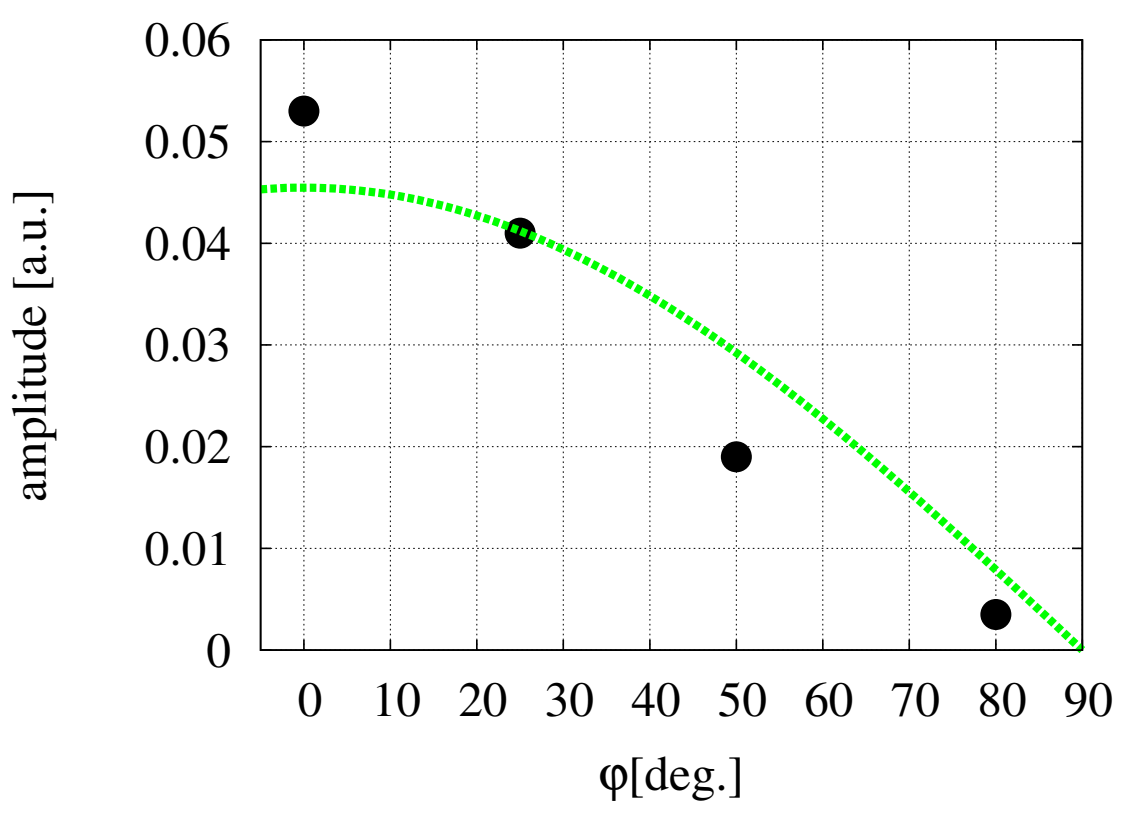

Figure 4: Polarization characteristics. $\varphi$ is an angle of the direction of the antenna element in a plane perpendicular to the axis connecting the antenna from the beam window. The dashed line is the cosine function.

had a wide irradiation room. Using a beam of Helium particles with an energy $6 \mathrm{MeV} / \mathrm{u}$ (time duration $700 \mu \mathrm{s}$, current value $550 \mu \mathrm{A}$, beam diameter $\sim 2 \mathrm{~cm}$ ) and three commercially available antennas (VHF antenna (76 - $90 \mathrm{MHz}$ ), UHF antenna (470 - $710 \mathrm{MHz})$ and broadband antenna (0.5 - $200 \mathrm{MHz})$ ), we tried to observe radio waves radiated in the forward direction as shown Fig. 5. Using a trigger signal of the beam, the signal were recorded with a real time spectrum analyzer that can measure transient signals as short as $100 \mu \mathrm{s}$.

\section{$\underline{\text { Results }}$}
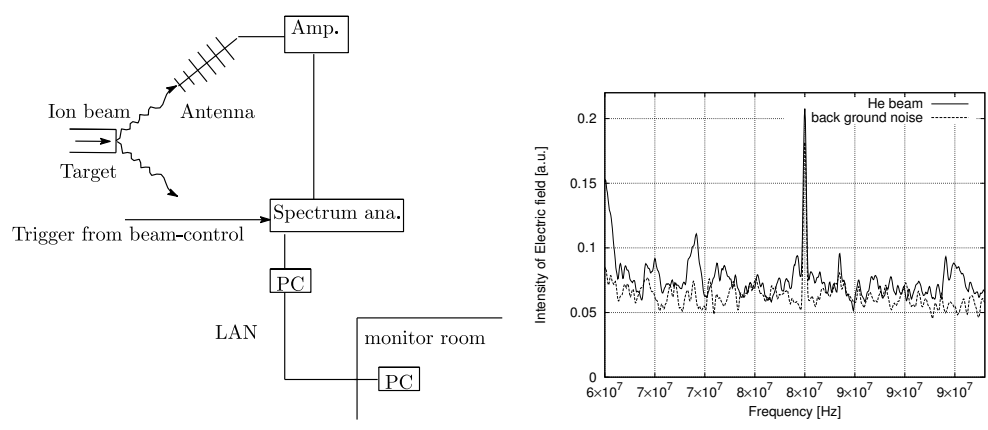

Figure 5: The left diagram shows the setup of the measurement, and the right diagram shows the intensity of the electric field measured using a VHF antenna. The solid line is the signal intensity due to beam irradiation, and the dotted line is the background intensity.

Fig. 5 shows the intensity of electric field measured using a VHF antenna which is tilted $35^{\circ}$ with 
respect to the beam axis. It can be seen that the electric field signal is slightly stronger than the background noise. The integrated value in the frequency range of $60-100 \mathrm{MHz}$ was about $16 \%$ stronger than the background. Furthermore, a signal strength of $35 \%$ was above background also observed when radio waves were measured in the same band using a broadband antenna. When the antenna was inclined $90^{\circ}$, the signal strength was about $3.5 \%$ above background.

\section{Summary}

The characteristics of radio waves generated by transition radiation were investigated using a particle accelerator and a radio antenna. The radio waves generated when electrons with an energy of $4.7 \mathrm{MeV}$ cross air from a titanium film were measured using a dipole antenna with a bandwidth of 800 to $880 \mathrm{MHz}$. The measured intensity of the pulse agreed with the theoretical value to within one order. The electric field strength was found to have a large angular dependence, and it was confirmed that the radio waves were polarized waves oscillating in a plane containing the beam axis and the wave number vector. We tried to measure transition radio waves with helium ion and directional antennas with bandwidth up to several hundred MHz. The signal range from about $15 \%$ to about $35 \%$ above background. In the future, it is necessary to conduct experiments with higher accuracy to investigate the characteristics of radio wave by the transition radiation process in detail.

\section{Acknowledgment}

The experiment with GHz-band antenna was performed using the compact electron accelerator system of Waseda Research Institute for Science and Engineering. The experiment with wide-band antennas was performed as part of the Research Project with Heavy Ions at NIRS-HIMAC.

\section{References}

[1] Allison, P. et al., Phys. Rev. D , 93, 082003 (2016)

[2] F.Heino, ASP Conference Series, vol. 395, 271 (2008)

[3] E. M. Holt, Journal of Physics: Conference Series 718, 052019 (2016)

[4] V.L.Ginzburg and I.M.Frank, Journ. Phys. USSR 9, 353-362 (1945)

[5] V. L. Ginzburg and V. N. Tsytovich, "Transition Radiation and Transition Scattering" , CRC Press ( 1990)

[6] D. John, Phys. Rev. D, 3, 2652 (1971)

[7] K.Sakaue et al., Proceedings of EPAC 2004, 2668 (2004) 